\title{
Research on Simulation and Survivability Measurement of Military Logistics Supply Network
}

\author{
WANG Tie-ning ${ }^{1, a}$, YU Shuang-shuang ${ }^{1, b}$, LIANG Bo ${ }^{1, c}$ and LI Ning ${ }^{2, d}$ \\ ${ }^{1}$ Academy of Armored Force Engineering, Beijing, China \\ ${ }^{2}$ Unit 78416 of PLA, Chongqing, China \\ aquingyueyingm@126.com, yuamanda@126.com, 'lannimi@sina.com, dxflnln@gmail.com
}

Keywords: computer simulation; military logistic; supply network; survivability measurement

\begin{abstract}
The military logistics supply network is generated through computer simulation firstly. Then on the basis of the military logistic network, the survivability index and measurement model of the military logistic network is established in topology layer and business layer, providing foundation and basis for the simulation experiment and survivability measurement of the actual military logistic network.
\end{abstract}

\section{Introduction}

Military logistics supply network bears the task of transporting military material from warehouse or factory to the troops. Military logistics supply network is complex with many nodes and edges. The nodes consist of rear warehouse, troop warehouse and transportation hubs such as crossings, bridges, ports, etc.; the edges of the network include various roads in different grades, railways, waterways and air transport, each of them has different distance. Its traffic capacity and external environment can affect the network traffic easily.

In future wars, both battle sides will pay more attention to break the enemy's support system and protect its own support system in making use of strategic military tactics ${ }^{[1]}$. Foreign scholar Lawrence and Daskin point out that considering facilities invalidation when designing the supply chain can obviously improve its service effect when the supply chain is interrupted ${ }^{[2,3]}$. This shows that, when designing military logistic network we should consider its capability facing the network invalidation in future wars. Studying on the survivability of military logistic network and raising its existence ability under an emergency condition becomes an important topic of new military period. The scholars aimed at different networks (military network ${ }^{[4]}$, communication network ${ }^{[5]}$, logistics network ${ }^{[6,7]}$ etc.) and studied their survivability and conception. But researches on the construction and survivability of military logistics network are scarce and no specific.

To explore the military logistics supply network survivability performance, a general logistics network based on the supply mode is generated by simulation method, then the actual supply business in the network is studied when the nodes, edges or vehicles of the network is disrupted by external factors such as natural disasters, enemy fire, etc. The military logistics support network can maintain the stability of its topology structure and the sustainability of its supply business on the basis of its topology attributes and emergency plans.

\section{Construction Simulation of Military Logistics Supply Network}

Typically, describing the structure of a network starts from two aspects: nodes and edges. Network node represents the connection between material supply lines in the military logistics supply network, namely the beginning and the end of the supply line. All the support entities, transportation hubs and supply objects are network nodes. According to the property of supply tasks, the nodes in the network are grouped into three types: supply node, connection node and demand point.

(1) Supply node. In the network, the node having the ability to receive and send material is called supply node, including strategic supply centers, regional supply centers and group supply centers. 
(2) Connection node. All the nodes that can't send or receive material are called connection nodes. They are applied to transport resources as a transit point mainly referring to the important railway stations, ports and other transportation hubs.

(3) Demand point. Material consumption units are the demand points.

Edge is essentially the abstract road network in the supply network. Each node of the network is connected by road, railway, waterway, airway, etc. together forming a road network carrier for the supply material delivery.

To study the performance and changes of the military logistics supply network, firstly the appropriate network model is set up, including its nodes, edges and the relationship between them.

\section{Generation Algorithm of Military Logistics Supply Network}

The skeleton of military logistics supply network is the supply centers and demand points at different level. They constitute the backbone network relying on organizational system supply relationship among the nodes. The military logistics supply network has a typical tree structure, of which the top is a strategic supply center, the bottom are the forces demand points, and in the middle are regional supply centers and group supply points. The backbone network reflects the relationship between major nodes of the supply network. In the actual supply network, in addition to supply node and demand point, there is connection node, such as common stations, docks and other transportation hubs. Meanwhile, there is not only organizational system step-by-step supply mode in the military logistics supply network, but also allocation supply mode and emergency supply mode. As a result, the topology structure of the supply network is not completely a tree structure, there is crossing between network nodes. Furthermore, the connection node belongs to public service node, it can be used by multiple supply chains in common, which is also an important cause for the cross structure of the network. The generation algorithm is described as follows.

(1) Initialization. The amount of strategic supply centers, regional supply centers, group supply centers, demand points, and connection nodes is $N_{1}, N_{2}, N_{3}, N_{4}, N_{5}$ respectively, the connection radius is $R$. Depending on the amount of various types of nodes, give each node a number, the rule is: strategic supply center, regional supply center, group supply center, demand point and connection node is numbered with L1-, L2-, L3-, L4-, L5- as beginning, followed by 1 to $N$ respectively. For example, number L1-1 represents the $1^{\text {st }}$ strategic supply center.

(2) Generation backbone network. 1) The military demand point and the group supply center acts as service recipient and service provider in the network to establish organizational relationship between them. 2) The group supply center and the regional supply center acts as service recipient and service provider in the network to establish organizational relationship between them. 3) The regional supply center and the strategic supply center acts as service recipient and service provider in the network to establish organizational relationship between them.

(3) Add connection nodes. Randomly generated $N_{5}$ connection nodes, these nodes represent train stations, docks and some other transportation hubs.

(4) Gridding. Circulate each connection node, designate a circle with the current connection node as the center, and $R$ is its radius. All nodes in the circular area are connected with the current connection node.

Here we assume that as long as two nodes of the network is connected by connection nodes, they can achieve the relation of supplier and supplied between them, that is, through gridding we actually simulate the organizational supply, allocation supply and skipping supply. Meanwhile, we can control the range of allocation supply and skipping supply in the network by adjusting the size of $R$.

\section{Generation Simulation of Military Logistics Supply Network}

This network generation simulation algorithm is implemented in VS2005 platform with C\# language. The effectiveness of the algorithm will be demonstrated by an actual network.

1) Description of Simulation Data

There are 122 military material demand points, a national road and two railway lines in a region. Others are mainly provincial, county and township roads. There are totally 58 counties and main 
towns in the region, which constitute the regional transportation hub of the road network. According to the situation, the original parameters related to the network are designed, as shown in Table 1.

Table 1. Parameters of military logistic network

\begin{tabular}{ccc}
\hline parameter name & parameter symbol & parameter value \\
\hline amount of strategic supply center nodes & $N_{1}$ & 1 \\
amount of regional supply center nodes & $N_{2}$ & 8 \\
amount of group supply center nodes & $N_{3}$ & 25 \\
military demand points & $N_{4}$ & 122 \\
connection nodes & $N_{5}$ & 58 \\
connection radius & $R$ & 10 \\
\hline
\end{tabular}

\section{2) Generated Supply Network with Simulation}

In order to make the computer-generated network diagram both authentic and aesthetic, the strategic supply centers, regional supply centers, group supply centers and military demand points are arranged in a concentric circle from inside to outside, radiating outward on the whole. Connection nodes are generated randomly in the area, and the other nodes are connected by the connection node and the connection radius to establish road network connection relationship. The generation process of supply network is as shown in Figure 1.

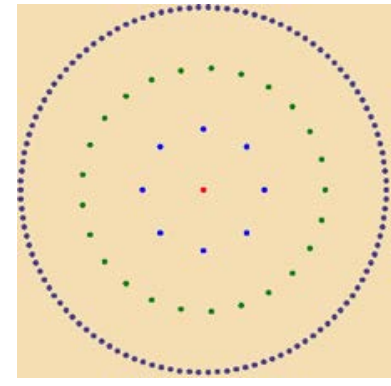

Figure 1(a). Distribution of supply center and military demand points

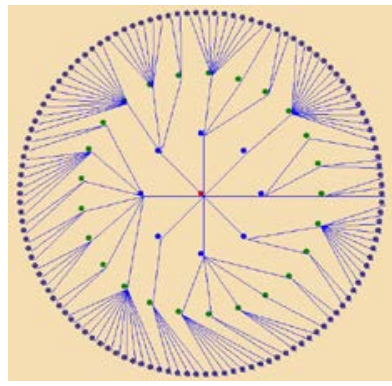

Figure 1(b). Organizational supply relationship

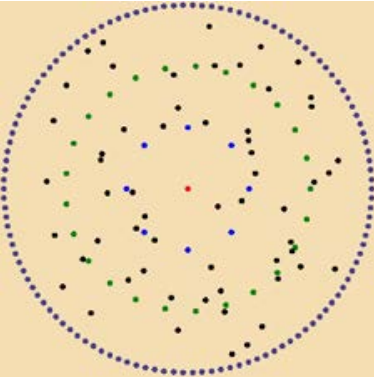

Figure 1(c). Node distribution diagram after adding the connection node

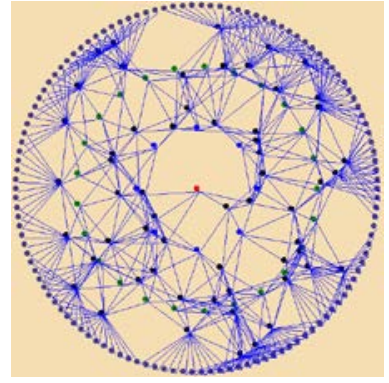

Figure 1(d). Final military logistic supply network model

Figure 1(a) shows the distribution of material resources at all levels and demand points, in which the point of the innermost layer is on behalf of the strategic supply center, the 8 points of the second layer is representative of regional supply centers, the 25 points of the third layer represents group supply centers, and the 122 points of the outermost layer represents military demand points. Figure 1(b) shows the organizational relationship between the supply nodes at all levels, is a typical tree structure, and the edge of the network is on behalf of supply relationship. Figure 1(c) is the node distribution diagram after adding connection nodes randomly based on Figure 1(a), the nodes in the figure represent the actual transportation hubs (such as counties) in the road network. Figure 1(d) is the gridding result through the connection radius $R$ based on Figure 1(c), which simulates the actual road network and the organizational supply, allocation supply and skipping supply relationship among the nodes in the network. The nodes of the military supply network consist of the supply centers at all levels, demand points and connection nodes, the edges of the network represent actual traffic roads (such as roads, railways and so on) in the road network.

Based on actual military supply, different military logistics supply networks with different complexity can be simulated and generated by changing the network parameters in Table 1.

\section{Survivability Measurement of Military Logistics Supply Network}

Survivability measurement is the metric to measure the size of the network survivability. Network survivability refers to the ability of the network system to complete the critical mission still timely when it suffers the attack, failures and accidents ${ }^{[8]}$. Survivability can be grouped into static survivability and dynamic survivability. Static survivability refers to research on the relevant characteristics of the networks while removing some nodes or edges of the network in accordance with certain strategies, there won't be coupling effects in these fault nodes, but the overall topology 
and performance of the network will suffer a great influence. Dynamic survivability is to study the network-related characteristics from a dynamic point of view. The survivability studied in this paper refers to static survivability.

\section{Research Mentality of Military Logistics Supply Network Survivability Measurement}

Military logistics supply network is a business network; its construction purpose is to finish the military supply tasks through the network's nodes and edges. Meanwhile, the military logistics supply network is a physical network, its nodes and edges are all real. The business network is the supply network's application layer, the physical network is the supply network's support layer, the business network runs on the physical network, the physical network supports the business network, and the physical network's survivability has a significant effect in business network.

To research the survivability measurement of military logistics supply network systematically and completely, we should study its survivability both in physical layer and business layer. To study the physical layer survivability, we mainly focus on its own characteristics in terms of topology without considering the business. To study the business layer survivability, we should design the survivability measurement indicators based on network's main function and troops demand. The network with good topology has a higher performance survivability and could adapt to different network environment: the network could provide the most efficient service in good network environment and keep the basic connectivity with good robustness in bad environment. Therefore, we choose the network efficiency and network robustness as the network topology survivability measurement indicators. Because of the supply network's military characteristics, we choose material arrival rate and user waiting time as the business survivability indicators, and they can reflect the troop's demands for the supply network in terms of quantify and time.

\section{Survivability Measurement of Network Topology Layer}

1) Network Robustness

Robustness is used to characterize the control system's insensitiveness with the perturbation of properties and parameters. In this paper, we define the robustness of military logistics supply network as follows:

Definition 1 Network Robustness. Network robustness $\eta_{R}$ is used to measure the average influence to the connectivity of the network with any nodes removed, the average ratio of connected nodes after and before any node being removed, namely:

$$
\eta_{R}=\frac{1}{n(n-1)} \sum_{i \in G_{k}} \sum_{j \neq i \in G_{k}} l_{i j}
$$

$G_{k}$ is the collection of the network nodes removed any node, $l_{i j}$ is the connectivity parameter, if there is a path between node $i$ and node $j, l_{i j}=1$, else $l_{i j}=0$.

Network Robustness $\left(\eta_{R}\right)$ describes the connectivity of the network removed any nodes, $\eta_{R}$ is $[0,1]$, the smaller value means the more disconnected nodes and the more segmentation of the network.

2) Network Efficiency

When the network is in heavy load or part of network doesn't work, some nodes or edges are blocked; the supplies in transit would be carried in other way to finish the supply task, thereby reducing efficiency of network supply transport. When a lot of nodes and edges are blocked, the supply network is divided into some sub-networks, the connectivity between nodes is destroyed, and there would be no arrival path. The transport efficiency of support network with good survivability would change in an acceptable range when a node or an edge is blocked, or else the supply network has a low survivability. Therefore, we define network efficiency to measure its ability to deal with the problem of transfer efficiency decreasing because of heavy load or part of the network doesn't work.

Definition 2 Network Efficiency $\left(\eta_{E}\right)$. We define the whole network efficiency as the average of the sum of the reciprocal of any two nodes' shortest distance. 


$$
\eta_{E}=\frac{1}{n} \sum_{i \in N} \sum_{j \in N} \frac{1}{d_{i j}}=\frac{2}{n(n-1)} \sum_{i \geq j}^{n} \frac{1}{d_{i j}}
$$

Network efficiency reflects the connectivity of the network; it could characterize the survivability of the global network's topology. The lager $\eta_{E}$ means network's larger connectivity and higher efficiency. We set $\eta_{E 0}$ as the normal efficiency of the network, then the reciprocal of the interrupt efficiency and the normal efficiency could reflect the sudden events' influence on network running.

$$
\xi=\frac{\eta_{E}}{\eta_{E 0}}
$$

\section{Survivability Measure of Supply Business Layer}

\section{1) Material Arrival Rate}

The fundamental purpose of military supply network is to meet the troops' material demand; therefore, the material arrival rate is the main method to measure the survivability of supply network service.

Definition 3 Material Arrival Rate $\left(\psi_{i}\right)$ of Demand Point $i$. we define the material arrival rate as the ratio of actual arrival quantity to demand quantity.

$$
\psi_{i}=\frac{Q}{Q_{0}}=\frac{Q_{0}-Q_{1}-Q_{2}}{Q_{0}}
$$

$Q_{0}$ is the original demand. If some nodes of the network are interrupted, some of the materials would be influenced. These materials can be of two types, first type could arrive through the other path; second type is isolated with the other network, these materials couldn't arrive in the most acceptable waiting time, represented with $Q_{2}$. Although the first type of supply could arrive at the demand point, the arrival time could be beyond the acceptable waiting time, we think it can't meet the demand point's requirement, represented with $Q_{1}$; the others which could arrive in acceptable waiting time could meet the demand. When calculating the arrival rate, we assume that the material is adequate to meet the requirement of demand point in the variety and quantity.

Obviously, the larger $\psi_{i}$ means the higher network survivability; it is essentially the measurable indicators of material loss in emergency.

Definition 4 Material Arrival Rate of the Network $(\psi)$. we define the whole network's material arrival rate as the average arrival rate of all demand points' arrival rate.

$$
\psi=\frac{\sum_{i \in N} \psi_{i}}{n}
$$

\section{2) User Waiting Time}

Considering supply network's military property, the network demand points have certain requests to the material arrival time, especially in emergency; user waiting time should be kept in a certain range. Therefore, we consider user waiting time as a measurable indicator, defined as follows.

Definition 5 the User Waiting Time of Demand Point $i\left(W T_{i}\right)$. we define $W T_{i}$ as the time it spends to get all the material from the time issuing the request, namely $W T_{i}=T_{\text {end }}-T_{\text {start }}$.

$T_{\text {end }}$ indicates the longest waiting time, when the network service interrupted, the original demands couldn't be satisfied.

Definition 6 Average Network User Waiting Time (WT ). we define the whole network's average user waiting time as the average value of all demand points' user waiting time.

$$
W T=\frac{W T_{i}}{n}
$$


Through observing the composition of each survivability index formula, it's easily to find out that the network robustness is easy to calculate for a given network, the network efficiency is just related to the shortest path. The shortest path could be calculated out by the Dijkstra method ${ }^{[9]}$. The calculation of supply business survivability index is more complex, it needs to design specialized algorithms. The corresponding algorithms refer to the article ${ }^{[10]}$.

\section{Summary}

This paper has studied the computer simulation models of the military logistics supply network and the measure method of its survivability. Firstly, we construct the model of military logistics supply network, which is the basis for further research; secondly, through dividing the supply network into physical layer and business layer, we construct the survivability indicators of topology layer and supply business layer, describe the arithmetic of these indicators. Based on this, we can work out the survivability simulation experiments through designing the network's attack strategy for the material supply network in battle environment in the future. At last we could come to the conclusion of military logistics supply network survivability.

\section{References}

[1] Wang Zongxi, Xu Dong. Military Logistics. Beijing: Tsinghua University Press (2003).

[2] Lawrence V S, Mark S D. Reliability models for facility location: The expected failure cost case. Transportation Science 39(3): 400-416(2005).

[3] Daskin M.S., E.H. Stern. A hierarchical objective set covering model for emergency medical service vehicle deployment. Transportation Science 15(2): 137-152(1981).

[4] Wen Qiaolin, Si Shoukui, Sun Xijing. Research on Survivability Index Function of Military Communication Network. System Security (6): 46-48(2010).

[5] Brostrom P, Holmberg K. Multi objective design of survivable IP networks. Ann Oper Res. 147: 235-253(2006).

[6] Wang Wei. Analysis and Study on the Survivability of Railway Network. Beijing: Beijing Jiaotong University (2011).

[7] Tan Gexin. Research on Complex Networks Topology Invulnerability Measurement. Nanjing: Nanjing University of Science and Technology (2011).

[8] Ellison R.J., Linger R.C., Longstaff T., Mead N. R. Survivable network system analysis: a case study. IEEE Software 16(4): 70-77(1999).

[9] Hu Yunquan, Guo Yaohuang. Operational Research Tutorial. Beijing: Tsinghua University Press (2003).

[10]Liang Bo. Research on Design and Survivability of Universal Equipment Materiel Support Network. Beijing: Academy of Armored Forces Engineering (2012). 